\title{
A new glimpse of FadR-DNA crosstalk revealed by deep dissection of the $E$. coli FadR regulatory protein
}

\author{
Yongchang Zhang ${ }^{1,2,3}$, Rongsui Gao ${ }^{2,3}$, Huiyan $\mathrm{Ye}^{2,3,4}$, Qingjing Wang ${ }^{2,3}$, Youjun Feng ${ }^{2,3 \bowtie}$ \\ ${ }^{1}$ College of Life Sciences, Nanjing Normal University, Nanjing 210023, China \\ 2 State Key Laboratory for Diagnosis and Treatment of Infectious Diseases, Collaborative Innovation Center for Diagnosis and \\ Treatment of Infectious Diseases, First Affiliated Hospital, Zhejiang University School of Medicine, Hangzhou 310003, China \\ ${ }^{3}$ Department of Medical Microbiology and Parasitology, Center for Infection and Immunity, Zhejiang University School of \\ Medicine, Hangzhou 310058, China \\ ${ }^{4}$ College of Life Science and Technology, Guangxi University, Nanning 530004, China \\ $\triangle$ Correspondence: fengyj@zju.edu.cn (Y. Feng)
}

Received August 6, 2014 Accepted August 29, 2014

\begin{abstract}
Escherichia coli (E. coli) FadR regulator plays dual roles in fatty acid metabolism, which not only represses the fatty acid degradation ( $f a d)$ system, but also activates the unsaturated fatty acid synthesis pathway. Earlier structural and biochemical studies of FadR protein have provided insights into interplay between FadR protein with its DNA target and/or ligand, while the missing knowledge gap (esp. residues with indirect roles in DNA binding) remains unclear. Here we report this case through deep mapping of old $E$. coli fadR mutants accumulated. Molecular dissection of $E$. coli K113 strain, a fadR mutant that can grow on decanoic acid (C10) as sole carbon sources unexpectedly revealed a single point mutation of T178G in fadR locus (W60G in FadR $\left._{\mathrm{k} 113}\right)$. We also observed that a single geneticallyrecessive mutation of W60G in FadR regulatory protein can lead to loss of its DNA-binding activity, and thereby impair all the regulatory roles in fatty acid metabolisms. Structural analyses of FadR protein indicated that the hydrophobic interaction amongst the three amino acids (W60, F74 and W75) is critical for its DNA-binding ability by maintaining the configuration of its neighboring two $\beta$-sheets. Further site-directed mutagenesis analyses demonstrated that the FadR mutants (F74G and/or
\end{abstract}

Electronic supplementary material The online version of this article (doi:10.1007/s13238-014-0107-3) contains supplementary material, which is available to authorized users.
W75G) do not exhibit the detected DNA-binding activity, validating above structural reasoning.

KEYWORDS FadR, fatty acid metabolism, crosstalk

\section{INTRODUCTION}

FadR, a member of the GntR super-family of transcription factors, is recognized as a global regulator (DiRusso et al., 1992, Henry \& Cronan, 1992, Clark \& Cronan, 2005). Not only does it exerts extensive effects on fatty acid metabolism (Clark, 1981, Nunn et al., 1983, Hughes et al., 1988), but also regulates acetate metabolism (icIR) (Gui et al., 1996, Maloy \& Nunn, 1981) and stress response (uspA) (Farewell et al., 1998). The dual functions of FadR regulatory protein in lipid metabolism can be described as follows: 1) repression of expression of genes essential for fatty acid degradation system, such as fadBA (DiRusso et al., 1993, Iram \& Cronan, 2006), fadH (He et al., 1997, Feng \& Cronan, 2010), fadM (Feng \& Cronan, 2009b), etc.; 2) activation of unsaturated fatty acid biosynthesis pathway via directly up-regulating the transcription of both fabA (Nunn et al., 1983, Henry \& Cronan, 1992, Campbell \& Cronan, 2001) and fabB (Campbell \& Cronan, 2001, Feng \& Cronan, 2009a). The physiological ligands of FadR regulator are long chain fatty acyl-CoA thioesters, which can neutralize and release FadR protein from its binding operators (Henry \& Cronan, 1992, Cronan, 1997). Thereby FadR protein can function as a sensor of the availability of long chain fatty acid in bacterial growth environment (Cronan \& Subrahmanyam, 1998, 

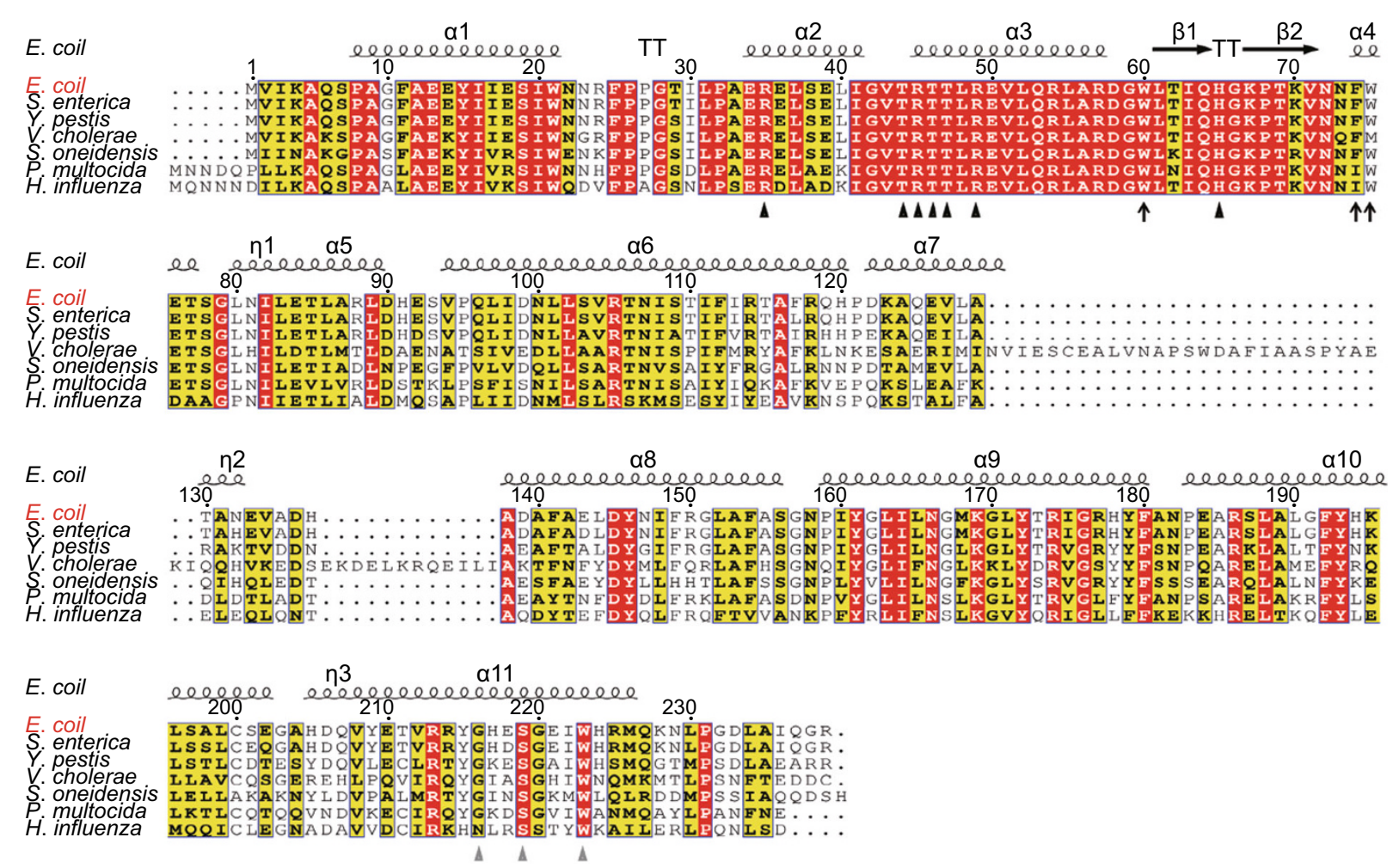

Figure 1. Sequence alignments of $E$. coli FadR regulatory protein with other homologues from six different species of Y-proteobacteria. The multiple alignments of amino acid sequences were conducted using ClustalW2 (http://www.ebi.ac.uk/Tools/ clustalw2/index.html), and the resultant output was processed by program ESPript 2.2 (http://espript.ibcp.fr/ESPript/cgi-bin/ESPript. cgi), generating the final BLAST photography (Feng \& Cronan, 2011b). Identical residues are in white letters with red background, similar residues are in black letters in yellow background, and the varied residues are in grey letters. As we earlier described (Feng et al., 2008), the protein secondary structure was shown in cartoon (on top), in terms of the structural architecture of $E$. coli FadR protein (PDB:1E2X) (van Aalten et al., 2000). $\alpha$ : alpha-helix; $\beta$ : beta-sheet; T: Turn; $\eta$ : coil. The DNA binding sites are indicated with black triangles (R35, T44, R45, T46, T47, R49 and 65H) (Xu et al., 2001), the ligand binding sites are shown with grey triangles (216G, 219S and 223W) (van Aalten et al., 2001), and the newly-proposed amino acids essential for DNA binding activity of FadR protein are highlighted with dark arrows (W60, F74 and W75). The FadR sequences are separately sampled from E. coli K12 (Accession no.: CAA30881), S. enterica (Salmonella enterica) (Accession no.: ACF63827), and Y. pestis (Yersinia pestis) (Accession no.: ZP_06207826), V. cholerae (Vibrio cholerae) (Accession no.: AAO37924), S. oneidensis (Shewanella oneidensis) (Accession no.: NP_718457), P. multocida (Pasterurella multocida) (Accession no.: AAK02132), and H. influenza (Haemophilus influenza) (Accession no.: AAC22085).

DiRusso et al., 1998, Campbell \& Cronan, 2001). The phenotype of $E$. coli that wild-type strains cannot grow on decanoic acid (C10) as sole carbon source (only the fadR mutant can) is due to the inability of short chain fatty acylCoA to turn off FadR-mediated repression of fad regulon (Iram \& Cronan, 2006).

It is well known that the fatty acid-responsive FadR regulatory protein is exclusively present in $\mathrm{Y}$-proteobacteria (Iram \& Cronan, 2005). The paradigm member of FadR regulator is the $E$. coli fadR protein product, a 239-residue polypeptide with solution structure of dimer (van Aalten et al., 2001, Xu et al., 2001, Feng \& Cronan, 2010), and also belongs to the helix-turn-helix type transcription factor, comprising $\mathrm{N}$-terminal DNA-binding domain and C-terminal ligand-binding domain (van Aalten et al., 2000, van Aalten et al., 2001, Xu et al., 2001). The accumulated crystal structures of FadR complexed with DNA target/acyl-CoA or alone have established structural basis for FadR-mediated transcription regulation of fatty acid metabolism (van Aalten et al., 2000, van Aalten et al., 2001, Xu et al., 2001). In addition to the crucial residues for ligand binding revealed by biochemical analyses (DiRusso et al., 1998), several amino acids essential for DNA binding have also been implied from FadR structural dissection (Xu et al., 2001). Additionally, the functional diversity has also been suggested by systematically biochemical documentation of a set of bacterial FadR homologues (Iram \& Cronan, 2005).

Although the FadR picture seems relatively complete right now, the residues other than direct DNA-binding sites remain unclear that contributes significantly to the binding activity of 


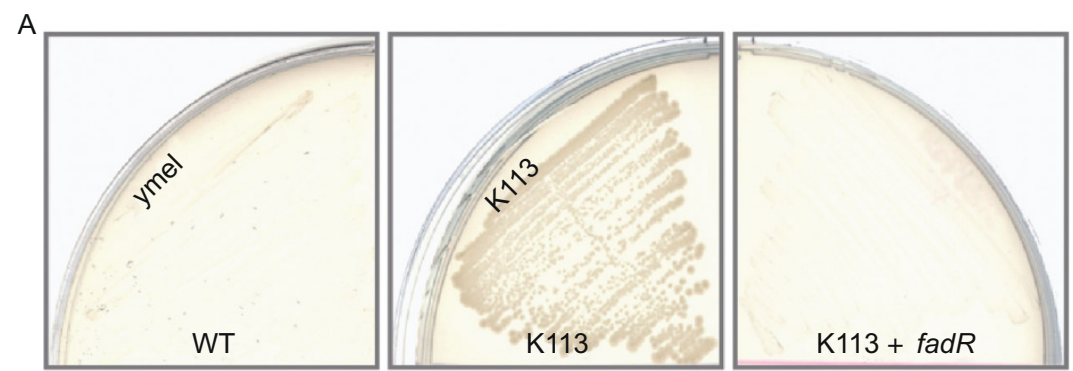

B

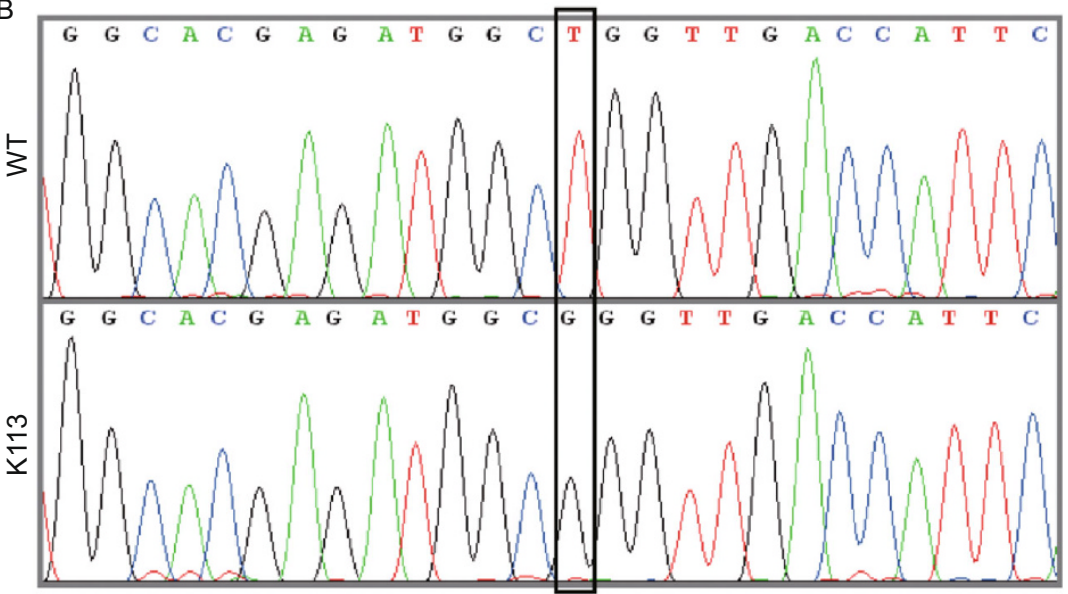

T178G (W60G)

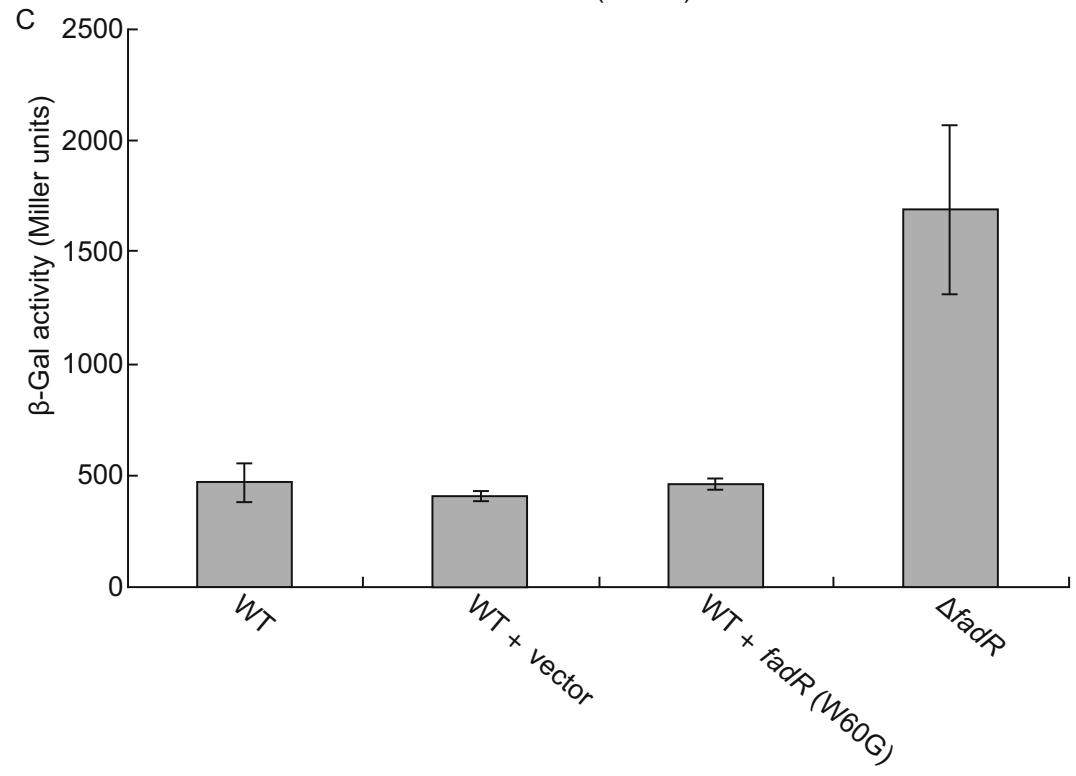

Figure 2. Determination of a single recessive mutation (W60G) present in fadR mutant strain K113. (A) Growth phenotype of E. coli fadR mutant strain $\mathrm{K} 113$ on minimal media with $\mathrm{C} 10$ as sole carbon source in comparison with those of the wild-type strain and the fadR complemented strain. The three strains used here included WT (ymel strain) (Feng \& Cronan, 2009a), K113 (fadR mutant) (Feng \& Cronan, 2009a, Clark et al., 1983) and the complemented strain FYJ7 (K113, zcf-117::Tn10, fadR ${ }^{+}$, Tet ${ }^{\mathrm{R}}$ ) (Feng \& Cronan, 2009a). (B) A single mutation (T178G) present in the K113 fadR revealed by direct DNA sequencing. The mutation of T178G at DNA level denotes the mutation of at protein level. (C) The mutation of W60G in FadR is genetically recessive. $\beta$-Gal activities were recorded in three independent experiments. All the strains assayed here contain a chromosomal fadBA-lacZ transcriptional fusion (Table 1). They are SI203 (WT), SI207 ( $\triangle$ fadR), FYJ225 (WT + vector) and FYJ226 (WT + fadR (W60G)), respectively. Vector: pBAD24. 
A
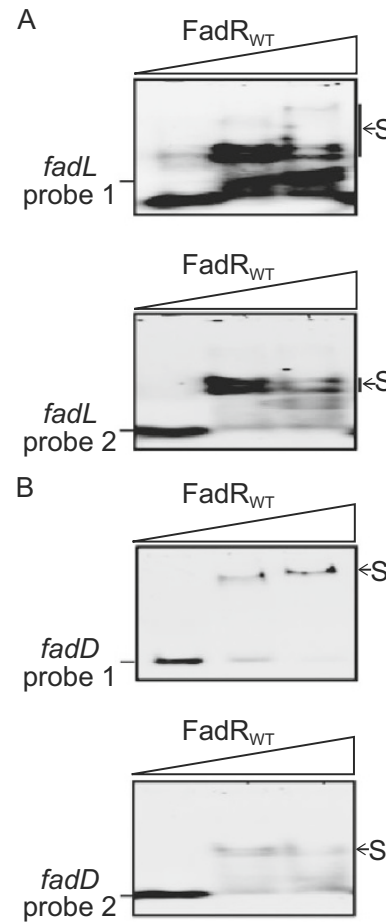

<Shift

probe
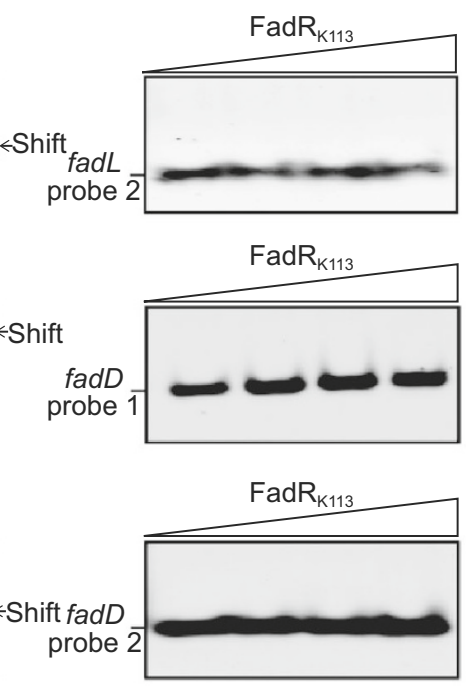

C
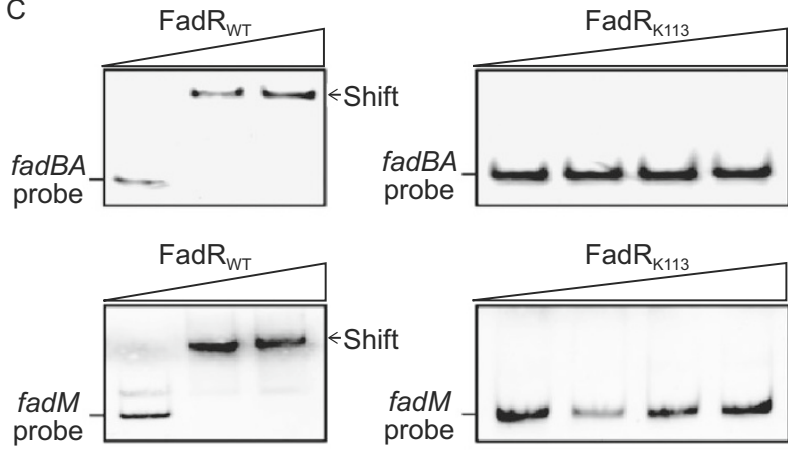

D
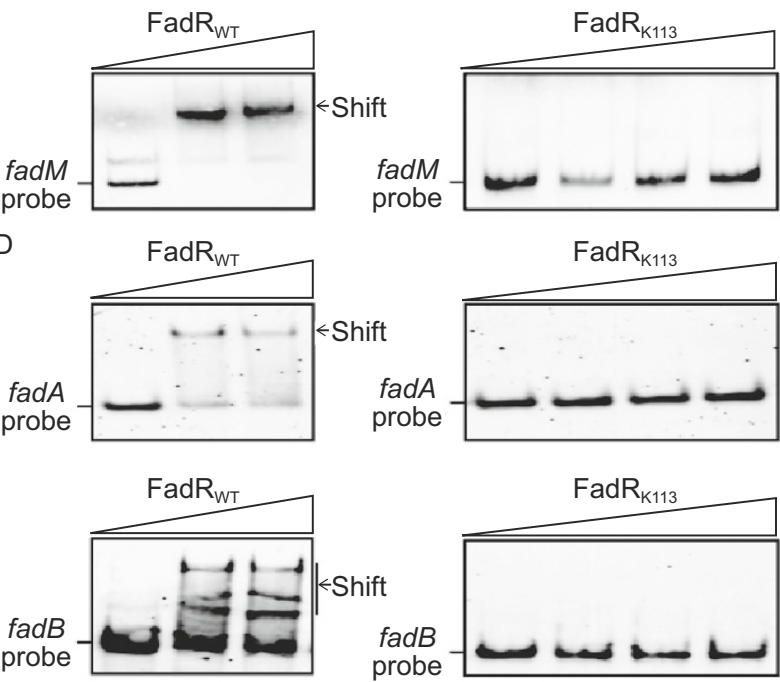

Figure 3. Mutant FadR protein $\left(F \operatorname{adR}_{\mathrm{K} 113}\right)$ losses its DNA binding activity in vitro. The two FadR-binding sites from both fadL (A) or fadD (B) can bind to the wild-type FadR protein (FadR $\mathrm{WT}_{\mathrm{T}}$ ), whereas not the mutant version of FadR, FadR $\mathrm{k}_{\mathrm{k} 113}(\mathrm{W60G})$. (C) The wild-type FadR protein (FadR $\mathrm{WT}_{\mathrm{WT}}$ ) can bind to promoter regions covering the FadR-binding sites of fadBA and fadM, whereas FadR $\mathrm{k}_{\mathrm{k} 113}$ does not. (D) FadR $\mathrm{k}_{\mathrm{k} 113}$ fails to bind to promoter regions of fabA and fabB, two UFA biosynthetic genes whereas FadR $\mathrm{WT}_{\mathrm{WT}}$ does. All the EMSA experiments were carried out using $7 \%$ native PAGE, and a representative result is shown here. In gel shift assays, FadR $R_{W T}$ is added as follows: $0,0.5$ and 1 pmol. Similarly, FadR $\mathrm{K} 113_{3}$ is supplemented in $0,0.5,1$ and 2 pmol, respectively. All the DIG-labeled probes are added to $0.1 \mathrm{pmol}$.

FadR to target promoters. Systematic analyses of $E$. coli fadR mutants collected in our laboratory in recent years allowed us to unexpectedly discover from K113 strain that a single point mutation (W60G) can impairs dramatically regulatory roles of FadR in lipid metabolism. Structure-guided mutagenesis analyses of FadR protein further suggested that the hydrophobic interaction amongst the three amino acids (W60, F74 and W75) might be essential for its DNAbinding ability by maintaining the configuration of its neighboring two $\beta$-sheets. Two more fadR super-repressor mutants were also discussed. The missing findings we reported in this paper might add new inputs into the picture of FadR-mediated regulation of bacterial lipid metabolism.

\section{RESULTS AND DISCUSSION}

Phenotypic and genetic dissection of the fadR mutant strain K113

FadR is a well-studied transcription factor that plays multiple roles in regulation of bacterial lipid metabolism. This type of regulator is consisted of a highly-conservative DNA-binding domain and a divergent acyl-CoA ligand-binding motif
(Fig. 1). Multiple sequence alignments of a set of FadR homologues revealed its pretty conservative architecture with an exception of $V$. cholerae FadR homologue in that it seemed likely to obtain an extra-inserting sequence of 40 residues (Fig. 1). Earlier biochemical analyses revealed three amino acids at C-terminus of FadR (G216, S219 and $223 \mathrm{~W}$ ) are critical for its binding activity to acyl-CoA ligand (DiRusso et al., 1998), whereas the reported crystal structures visualized six $\mathrm{N}$-terminal residues (R35, 44T, 45R, 46T, 47T, 49R and $65 \mathrm{H}$ ) in direct contact with its cognate DNA (van Aalten et al., 2000, Xu et al., 2001).

We had occasion to sequence the original fadR mutation that of strain K113 of Overath and coworkers, originally called dec-16 and now called fadR16. We found that the fadR16 lesion results in substitution of glycine for tryptophan 60 , a residue that plays no direct role in DNA binding. We showed that fadR16 ( adR $_{\text {K } 113}$ ) was recessive to the wildtype allele. As we knew that the wild-type E. coli strains can grow on media with long-chain fatty acids as sole carbon source, but not on short-chain fatty acids like decanoic acid (C10) (Fig. 2A). In contrast, the fadR mutants usually exhibited a phenotype with capability of growing on $\mathrm{C} 10$ media. Like most of the other fadR mutants, the K113 strain 
A
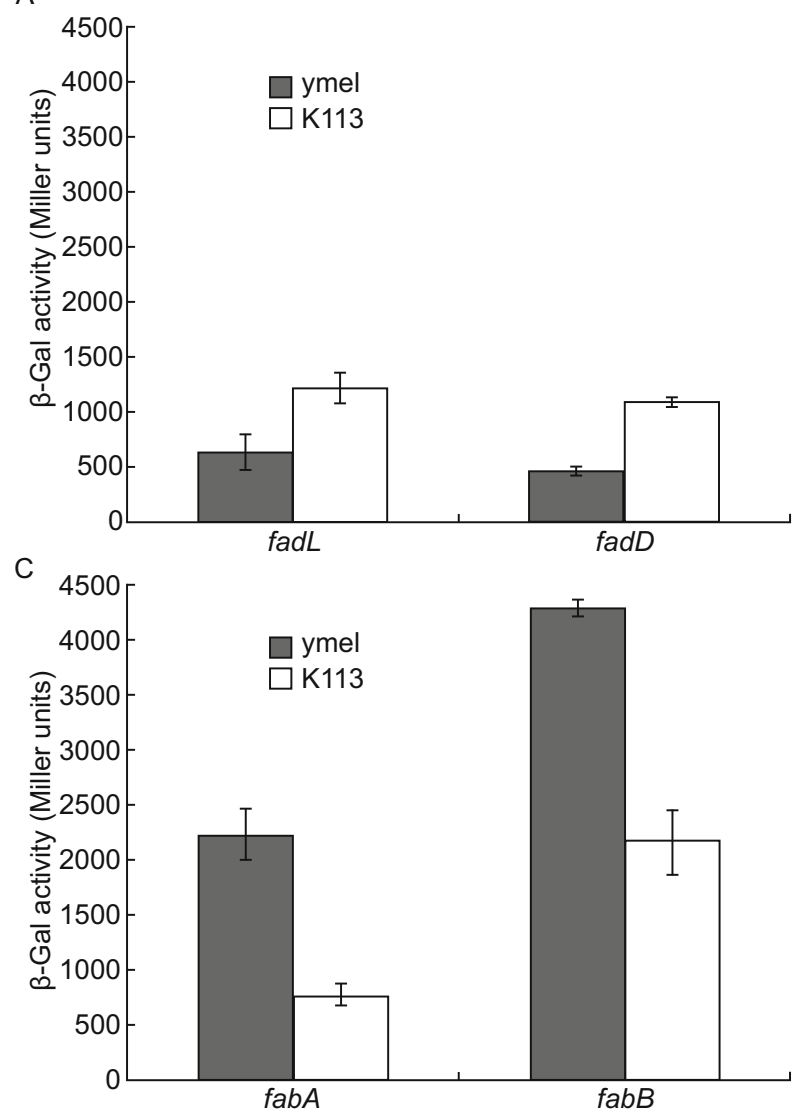

B

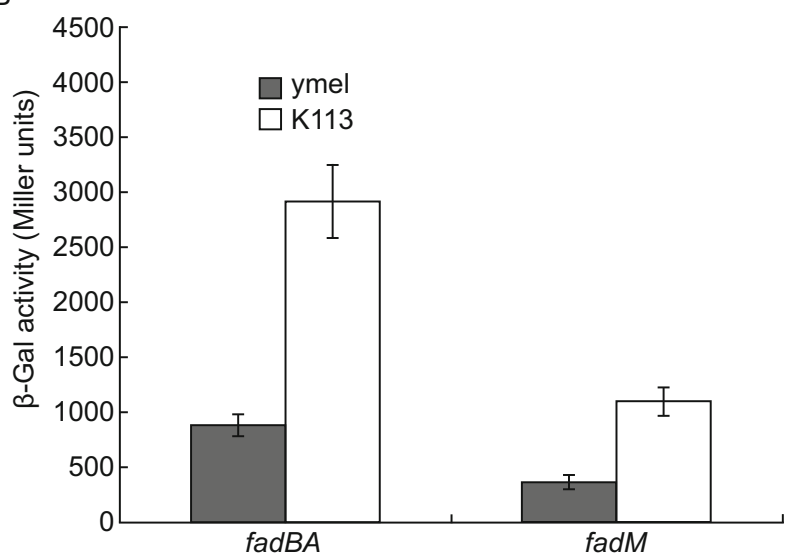

Figure 4. Regulatory dysfunction of fatty acid metabolism in K113 fadR strain. (A) Assays for transcriptional activities fadL and $f a d D$, two genes of fatty acid transport system in the fadR strain K113. (B) Comparative analyses of expression levels of fadBA, a major member of beta-oxidation system and fadM, an auxiliary player of fad system in the fadR strain $\mathrm{K} 113$ relative to those of the wild-type ymel. (C) Effects on transcriptional levels of $f a b A$ and $f a b B$, two key genes required for UFA synthesis due to fadR mutation in K113 strain. E. coli strains were grown in RB liquid media. $\beta$-Gal activities from three independent experiments are expressed in average \pm standard deviations. In panel A, the strains used were FYJ185 (ymel, fadL-lacZ fusion), FYJ186 (K113, fadL-lacZ fusion), FYJ183 (ymel, fadD-lacZ fusion) and FYJ184 (K113, fadD-lacZ fusion). In panel B, the four strains included FYJ34 (ymel, fadBA-lacZ fusion), FYJ38 (K113, fadBA-lacZ fusion), FYJ35 (ymel, fadM-lacZ fusion) and FYJ39 (K113, fadM-lacZ fusion). In panel C, the strains tested referred to FYJ36 (ymel, fabA-lacZ fusion), FYJ 40 (K113, fabA-lacZ fusion), FYJ37 (ymel, fabB-lacZ fusion), FYJ41 (K113, fabB-lacZ fusion), respectively.

can grow on $\mathrm{C} 10$ plates (Fig. 2A), and this fadR phenotype can be restored to that of the parental strain ymel upon functional complementation of a copy of wild-type fadR gene (Fig. 2A). Comparative analyses of direct DNA sequencing results dissected firstly that this fadR phenotype is only attributed to the presence of a point mutation of T178G (W60G) in K113 strain (Fig. 2B).

Monitoring $\beta$-gal activity of a transcriptional fadBA-lacZ fusion revealed that removal of fad $R$ led to $>3$-fold increment of expression level, whereas the introduction of a plasmid pBAD-borne mutant fadR ( $\left.f a d R_{\mathrm{K} 113}\right)$ into the wild-type strain could not result in any significant change of fadBA transcription (Fig. 2C). It was anticipated that $\mathrm{FadR}_{\mathrm{K} 113}$ with mutation of W60G cannot compete with the wild-type version of FadR in the context of physiological interplay between FadR and fadBA promoter, indicating that the W60G mutation is genetically-recessive.

\section{Functional impairment of FadR regulatory roles by a genetically recessive single mutation of W60G}

To fully address the effect of the genetically-recessive single mutation of W60G on regulatory function of FadR protein, we employed the in vitro and in vivo approaches including EMSA and transcriptional fusion. The DNA binding activity of the mutant FadR protein $\left(\mathrm{FadR}_{\mathrm{K} 113}\right)$ was examined in vitro using gel shift assays. Eight sets of DIG-labeled DNA probes were prepared. Among them, two probes were designed for 
A

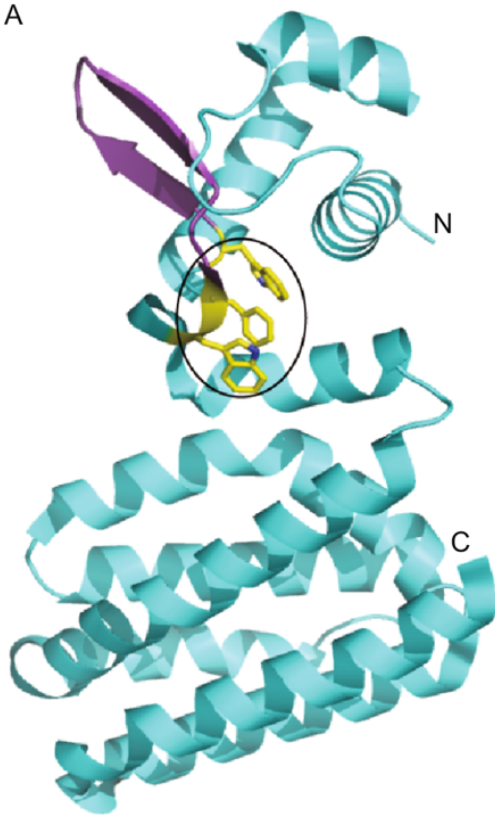

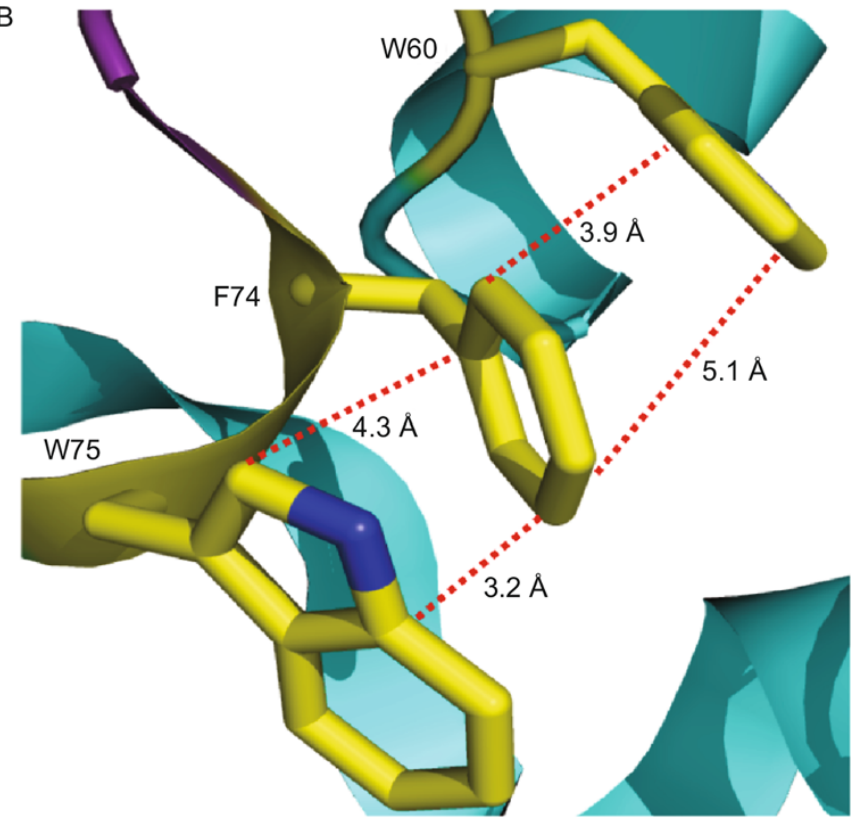

Figure 5. Structural analyses of FadR protein suggest that a hydrophobic interaction amongst W60, F74 and W75 might be important for its DNA binding activity. (A) Ribbon structure of $E$. coli FadR in monomer. N: N-terminus; C: C-terminus. $\alpha$-Helix is blue, $\beta$-sheet is purple, and the three amino acids (W60, F74 and W75) that were proposed in this study is highlighted with a circle. (B) The enlarged view of the three critical residues (W60, F74 and W75). The hydrophobic bonds are expressed with dotted red lines, and the instance between two relevant atoms is labeled (angstrom). The photography was generated by Pmol software using the crystal structure's PDB file of FadR protein (PDB: 1E2X).

either fadL or fadD in that they have two FadR-binding palindromes (fad $L$ probe1 plus fad $L$ probe 2 (Fig. $3 A$ ) and fadD probe1 plus fadD probe 2 (Fig. 3B)) (Feng \& Cronan, 2012). As we expected, the wild-type FadR protein ( $\left.F a d R_{W T}\right)$ exhibited obvious binding to fadL ( $f a d D$ ) promoter (Fig. 3A and $3 \mathrm{~B}$ ), however the $\mathrm{FadR}_{\mathrm{K} 113}$ protein was not visualized to interact with them at the same protein level used in our trials (no more than $10 \mathrm{pmol}$ ). The promoters of fadBA (Feng \& Cronan, 2012) and fadM (Feng \& Cronan, 2009b), two members of $\beta$-oxidation system, also cannot be bound by FadR $_{\mathrm{K} 113}$ protein (Fig. $3 \mathrm{C}$ ). The similar scenario was also observed with fabA and fabB (Feng \& Cronan, 2011a), two important genes required for UFA biosynthetic pathway (Fig. 3D).

To further prove the dysfunction of $\mathrm{FadR}_{\mathrm{K} 113}$ in vivo, we evaluated altered expression profile of a series of FadRregulated genes in K113 strain vs. ymel strain (parental strain). As a result, we noted that 1 ) about 2-fold improved expression of both fadL and fadD, two genes encoding LCFA transport system in K113 strain over ymel strain (Fig. 4A), 2) 3.5-fold increment of fadBA expression and 2.5-fold elevated transcription of fadM in $\mathrm{K} 113$ strain relative to ymel strain (Fig. 4B), and 3) 2 to 2.5 -fold decreased expression of fabA and $f a b B$ (Fig. $4 C$ ). The combined evidence demonstrated that functional impairment of fad $R_{\mathrm{K} 113}$ regulatory roles in lipid metabolism is due to a genetically-recessive single mutation of W60G in FadR protein.

\section{A tripartite hydrophobic interaction required for DNA binding activity by FadR}

Earlier structural investigations illustrated the overall architecture of E. coli FadR complex and its target DNA (Fig. S1) (Xu et al., 2001). The integrated evidence from structural modeling combined with genetic/biochemical data showed that the residue W60 is not in direct contact with DNA (Fig. 5A), but rather forms an aromatic cluster with two residues phenylalanine 74 and tryptophan 75 (F74 and W75). The measured distances amongst the three aromatic side chains varied from $3.2 \AA$ to $5.1 \AA$ (Fig. $5 B$ ) well positioned for hydrophobic bonding interactions in that it fits generally the criteria for defining a hydrophobic bond (interaction). We ambitiously hypothesize that the above hydrophobic bonds are necessary to maintain the flexibility of the two neighboring $\beta$-sheets, which in turn stabilizes configuration of its DNA binding domain (Fig. 5A). If so, mutation of any one of the three residues might well result in the loss of DNA binding activity.

To test this possibility, we prepared three more mutant FadR proteins (F74G, W75G and F74G plus W75G) using an approach of site-directed mutagenesis. In addition to the two versions of FadR protein (FadR $\mathrm{FT}_{\mathrm{WT}}$ and $\mathrm{FadR}_{\mathrm{K} 113}$ (W60G)), we also purified the other three types of mutant FadR proteins to homogeneity (Fig. 6A). Somewhat different from that of the recombinant FadR $R_{W T}$ in E. coli expression 
A
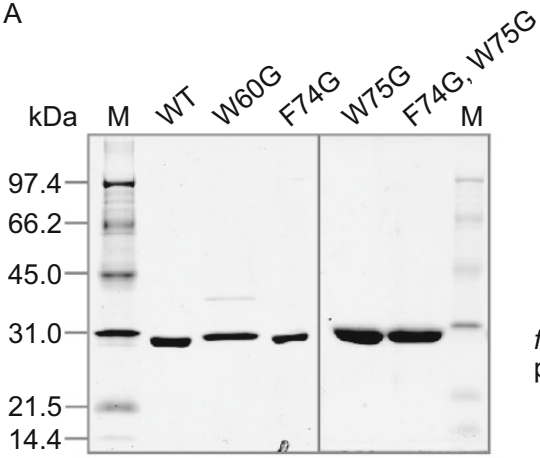

D

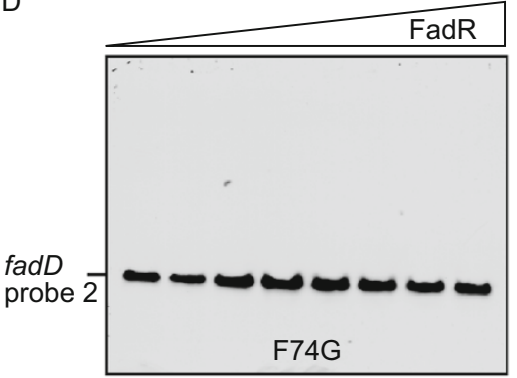

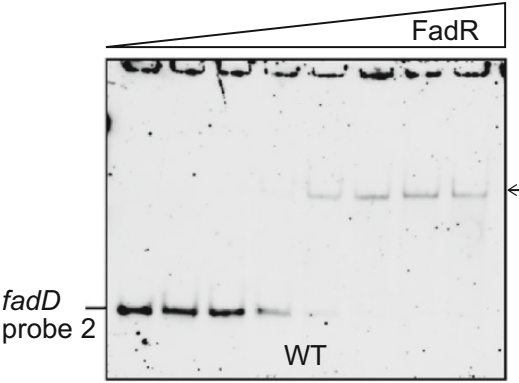

E

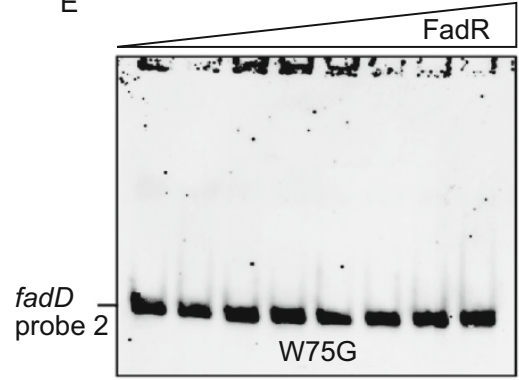

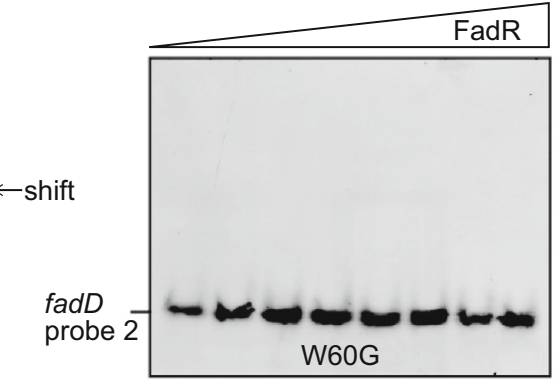

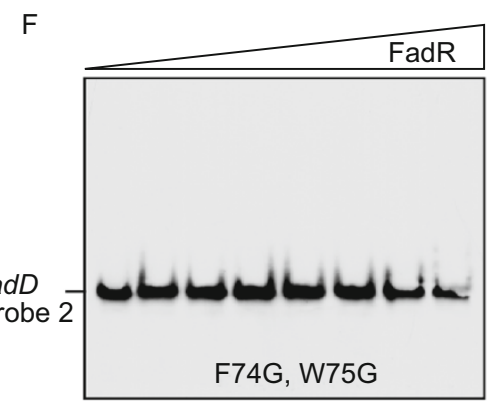

Figure 6. In vitro preparation and functional analyses of four mutant FadR proteins plus its wild-type version. (A) Expression and purification of four mutant FadR proteins and its wild-type. M: protein standard marker (Biorad). EMSA-based assays of binding

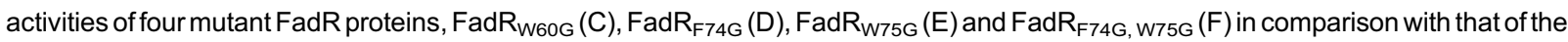
wild-type version (B). The gel shift tests were all conducted using $7.5 \%$ native PAGE, and a representative result is shown here. In these assays, FadR protein is added as follows: $0,0.05,0.1,0.5,1,2,5$ and 10 pmol. The DIG-labeled fadD probe 2 is added to 0.2 pmol.

system ( $>70 \%$ solubility), the solubility of all the other four mutant proteins is estimated to vary from $5 \%$ to $15 \%$ (not shown). The fact that all of the mutants were appreciably less soluble than the wild-type protein is consistent with exposure of hydrophobic groups. For convenient comparison, the DIG-labeled fadD probe 2 with relatively high affinity to FadR protein was used in gel shift assays. As we mentioned before, FadR $R_{W T}$ protein can bind to fadD probe 2 in a dose-dependent manner (Fig. 6B), whereas no significant binding of the mutant protein $\mathrm{FadR}_{\mathrm{K} 113}$ (W60G) was found (Fig. 6C). Similar to what observed with FadR $\mathrm{K}_{\mathrm{K} 113}$ mutant protein, other two mutant FadR proteins with single mutation of either F74G (Fig. 6D) or W75G (Fig. 6E) did not exhibit DNA binding activity, as well as the FadR mutant with the double point-mutations of F74G plus W75G (Fig. 6F). Given the above structure-guided site-directed mutational analyses, we concluded that the aromatic cluster plays a key, but indirect, role in DNA binding. The fact that all three residues are aromatic and our prior finding that naturally occurring FadR proteins having isoleucine in place of F74 bind DNA poorly suggested that $\pi$-interactions may play a role in cluster function (Xu et al., 2001). However, we found that the F74I version of $E$. coli FadR had full DNA binding activity which ruled out $\pi$-interactions. Hence, it seemed likely that the cluster stabilizes the configuration of its DNA binding domain (Fig. 5).

\section{CONCLUSIONS}

Although the K113 fadR strain has been known for years, we are first to describe systemically regulatory dysfunction of lipid metabolism. Somewhat it is unexpected to note that the molecular determinant is a genetically-recessive pointmutation of W60G in fadR locus (Figs. 2 and 5). Relative to the known residues with direct contacting DNA operates, this hydrophobic amino acid of tryptophan at the position 60 of FadR regulatory protein represent a new functional residue in that it is without close contact to its cognate DNA, but required for capability of DNA binding.

In addition, we addressed two more fadR super-repressor mutant strains (TH182 and $\mathrm{TH} 183$, featuring that the repression of fad regulon expression cannot be relieved by oleate). In general consistency with the report by Hughes et al. (Hughes et al., 1988), we also found the molecular determinants of two fadR super-repressor strains $\mathrm{TH} 182$ and $\mathrm{TH} 183$, each of which is a single point mutation localized in $\mathrm{C}$-terminal ligand binding domain (G638A, R213H in TH182 and G647A, G216E in TH183) (not shown). Ambitiously, we firstly proposed a model in which the hydrophobic interaction of W60 with other two aromatic amino acids (F74 and W75) is critical for the maintenance of configuration of the DNA binding domain of FadR protein at $\mathrm{N}$-terminus (Fig. 5). It seemed likely that our findings might supplement three more new functional residues of FadR regulator. 
Table 1. Strains and plasmids used in this study

\begin{tabular}{|c|c|c|}
\hline $\begin{array}{l}\text { Bacteria or } \\
\text { plasmids }\end{array}$ & Relevant characteristics & References/sources \\
\hline \multicolumn{3}{|l|}{ E. coli strains } \\
\hline BL21 (Tuner) & An expression host for recombinant plasmids & Lab stock \\
\hline MC4100 & $\mathrm{F}^{-}, \operatorname{araD} 139, \Delta(\operatorname{argF-lac}) 169$ & Feng \& Cronan (2009b) \\
\hline 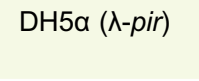 & $\Delta / a c$ host for $\mathrm{pAH} 125$ and its derivatives & $\begin{array}{l}\text { Haldimann \& Wanner (2001); Feng \& Cronan } \\
\text { (2009b); Feng \& Cronan (2009a) }\end{array}$ \\
\hline MC1061 & Wild-type of $E$. coli K-12, $\Delta / a c$ & Feng \& Cronan (2009b) \\
\hline MFH8 & UB1005, fadR::Tn10 & Henry \& Cronan (1992) \\
\hline ymel & Parental strain of $\mathrm{K} 113$ & Feng \& Cronan (2009a) \\
\hline K113 & ymel, fadR16 & Clark (1981) \\
\hline JT160 & MC1061, fabA-lacZ fusion, $\mathrm{Kan}^{\mathrm{R}}$ & Lab stock, Feng \& Cronan (2011a) \\
\hline JT161 & MC1061, fabB-lacZ fusion, $\mathrm{Kan}^{\mathrm{R}}$ & Lab stock, Feng \& Cronan (2011a) \\
\hline FYJ17 & MC1061, fadM-lacZ fusion, $\mathrm{Kan}^{\mathrm{R}}$ & Feng \& Cronan (2009b) \\
\hline FYJ30 & JT180, fadR::Tn10, $\operatorname{Kan}^{R}$, Tet ${ }^{R}$ & Feng \& Cronan (2011a) \\
\hline FYJ32 & JT181, fadR::Tn10, Kan ${ }^{R}, \operatorname{Tet}^{R}$ & Feng \& Cronan (2011a) \\
\hline FYJ34 & ymel, fadBA-lacZ fusion, $\mathrm{Kan}^{\mathrm{R}}$ & P1vir(SI203)xymel ${ }^{\mathrm{C}}$ \\
\hline FYJ35 & ymel, fadM-lacZ fusion, $\operatorname{Kan}^{R}$ & P1vir(FYJ17)xymel ${ }^{\mathrm{C}}$ \\
\hline FYJ36 & ymel, fabA-lacZ fusion, $\operatorname{Kan}^{R}$ & P1vir(JT160)xymel ${ }^{\mathrm{c}}$ \\
\hline FYJ37 & ymel, fabB-lacZ fusion, $\operatorname{Kan}^{R}$ & P1vir(JT161)xymel ${ }^{\mathrm{C}}$ \\
\hline FYJ38 & $\mathrm{K} 113$, fadBA-lacZ fusion, $\mathrm{Kan}^{\mathrm{R}}$ & P1vir(SI203)xK113 \\
\hline FYJ39 & $\mathrm{K} 113$, fadM-lacZ fusion, $\mathrm{Kan}^{\mathrm{R}}$ & P1vir(FYJ17)xK113 ${ }^{\mathrm{C}}$ \\
\hline FYJ40 & $\mathrm{K} 113$, fabA-lacZ fusion, $\mathrm{Kan}^{\mathrm{R}}$ & P1vir(JT160)xK113 ${ }^{\mathrm{C}}$ \\
\hline FYJ41 & $\mathrm{K} 113$, fabB-lacZ fusion, $\mathrm{Kan}^{\mathrm{R}}$ & P1vir(JT161)xK113 \\
\hline SI203 & fadBA-lacZ transcription fusion & Iram \& Cronan (2005); Feng \& Cronan (2010) \\
\hline SI207 & fadBA-lacZ transcription fusion, $\Delta$ fadR::Tn10 & Iram \& Cronan (2005); Feng \& Cronan (2010) \\
\hline FYJ104 & FYJ103, fadL-lacZ transcription fusion & This work \\
\hline FYJ158 & $\mathrm{DH} 5 \alpha$ ( $\lambda$-pir) carrying pAH-PfadD & Feng \& Cronan (2011a) \\
\hline FYJ159 & $\begin{array}{l}\text { MC4100, fadD-lacZ transcription fusion integrated into } \\
\text { the chromosomal } \lambda \text {-attB site }\end{array}$ & This work \\
\hline FYJ183 & ymel, fadD-lacZ transcriptional fusion & P1vir(FYJ159)×ymel ${ }^{\mathrm{c}}$ \\
\hline FYJ184 & $\mathrm{K} 113$, fadD-lacZ transcriptional fusion & 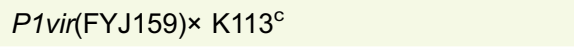 \\
\hline FYJ185 & ymel, fadL-lacZ transcriptional fusion & P1vir(FYJ104)×ymel ${ }^{\mathrm{c}}$ \\
\hline FYJ186 & $\mathrm{K} 113$, fadL-lacZ transcriptional fusion & P1vir(FYJ104)× K113 ${ }^{\mathrm{C}}$ \\
\hline FYJ187 & MC4100 carrying pINT-ts & Feng \& Cronan (2011a) \\
\hline FYJ191 & BL21(DE3) carrying pET28-fadRec(W75G) & This work \\
\hline FYJ192 & BL21(DE3) carrying pET28-fadR_K113 & This work \\
\hline FYJ206 & BL21(DE3) carrying pET28-fadRec(F74G) & This work \\
\hline FYJ207 & BL21(DE3) carrying pET28-fadRec(F74G/W75G) & This work \\
\hline FYJ221 & Topo 10 carrying pBAD-fadR_K113 & This work \\
\hline FYJ225 & SI203 carrying pBAD24 & This work \\
\hline FYJ226 & SI203 carrying pBAD-fadR_K113 & This work \\
\hline \multicolumn{3}{|l|}{ Plasmids } \\
\hline pET28(a) & Commercial T7-driven expression vector & Novagen \\
\hline
\end{tabular}


Table 1 continued

\begin{tabular}{|c|c|c|}
\hline $\begin{array}{l}\text { Bacteria or } \\
\text { plasmids }\end{array}$ & Relevant characteristics & References/sources \\
\hline pBAD24 & $\begin{array}{l}\text { Arabinose-inducible promoter-driven expression vector, } \\
\mathrm{Amp}^{\mathrm{R}}\end{array}$ & Guzman et al. (1995) \\
\hline pBAD-fadR_K113 & pBAD24 carrying $\mathrm{K} 113$ fadR gene, $\mathrm{Kan}^{\mathrm{R}}$ & This work \\
\hline pET28-fadRec & pET28 carrying E. coli fadR, $\operatorname{Kan}^{R}$ & Feng \& Cronan (2009b); Feng \& Cronan (2009a) \\
\hline pET28-fadR_K113 & pET28 carrying $\mathrm{K} 113$ fadR gene, $\mathrm{Kan}^{\mathrm{R}}$ & This work \\
\hline $\begin{array}{l}\text { pET28-fadRec } \\
(\text { F74G) }\end{array}$ & $\begin{array}{l}\text { pET28 carrying } E \text {. coli fadR gene with a single } F 74 G \\
\text { mutation, } \mathrm{Kan}^{\mathrm{R}}\end{array}$ & This work \\
\hline $\begin{array}{l}\text { pET28-fadRec } \\
\text { (W75G) }\end{array}$ & $\begin{array}{l}\text { pET28 carrying E. coli fadR gene with a single W75G } \\
\text { mutation, } \operatorname{Kan}^{\mathrm{R}}\end{array}$ & This work \\
\hline $\begin{array}{l}\text { pET28-fadRec } \\
\text { (F74G, W75G) }\end{array}$ & $\begin{array}{l}\text { pET28 carrying } E \text {. coli fadR gene with the double } \\
\text { mutations (F74G, W75G), } \operatorname{Kan}^{R}\end{array}$ & This work \\
\hline
\end{tabular}

a CGSC denotes Coli Genetic Stock Center, Yale University; ${ }^{\mathrm{b}}$ Selection for tetracycline resistance; ${ }^{\mathrm{c}}$ Selection for kanamycin resistance.

Together, we believed that the deep dissection of the E. coli FadR regulatory protein provided a new glimpse of FadR-DNA interplay/crosstalk in the context of lipid metabolism.

\section{MATERIALS AND METHODS}

Bacterial strains and growth conditions

All the strains used here are $E$. coli $\mathrm{K}-12$ derivatives (Table 1). Three types of media used for $E$. coli included LB medium (Luria-Bertani medium containing $10 \mathrm{~g}$ of tryptone, $5 \mathrm{~g}$ of yeast extract and $10 \mathrm{~g}$ of $\mathrm{NaCl}$ per liter), Rich broth (RB medium containing $10 \mathrm{~g}$ of tryptone, 1 $\mathrm{g}$ of yeast extract and $5 \mathrm{~g}$ of $\mathrm{NaCl}$ per liter) and minimal medium $\mathrm{M} 9$ (Iram \& Cronan, 2006) supplemented with $0.4 \%$ glucose, $0.1 \%$ vitamin-free Casamino Acids, $1 \mathrm{mmol} / \mathrm{L} \mathrm{MgSO}_{4}$ and $0.1 \mathrm{mmol} / \mathrm{L}$ $\mathrm{CaCl}_{2}$, and $0.001 \%$ thiamine were used for bacterial growth and analyses of $\beta$-galactosidase activity. If necessary, antibiotics were used as follows (in $\mathrm{mg} / \mathrm{liter}$ ): 100 for sodium ampicillin, 50 for kanamycin sulfate, 20 for chloramphenicol and 15 for tetracycline $\mathrm{HCl}$.

\section{Plasmids and DNA manipulations}

The expression vector pET28a (Novagen) was used for protein preparation (Table 2). The $\mathrm{K} 113$ fadR was amplified using primers fadRec-F2 plus fadRec-R2 that carried EcoRI and Sall restriction sites, respectively (Table 2 ). The PCR product was digested with these enzymes and inserted into vector pBAD24 (Guzman et al., 1995) cut with the same enzymes giving the plasmid pBADfadR_K113 (Table 1). All plasmids acquired were validated by PCR testing plus direct DNA sequencing.

\section{$P 1_{\text {vir }}$ phage transduction}

$P 1_{\text {vir }}$ transductions were carried out as described by Miller (Miller, 1992) with minor modifications. Strains FYJ34, FYJ35, FYJ36, FYJ37, FYJ183 and FYJ185 (Table 1) were obtained by $P 1_{\text {vir }}$ transduction of strain ymel, using lysates grown on strains SI203 (fadBA-lacZ), FYJ17 (fadM-lacZ), JT160 (fabA-lacZ), JT161 (fabBlacZ), FYJ159 (fadD-lacZ) or FYJ104 (fadL-lacZ) with selection for kanamycin resistance. Strains FYJ38, FYJ39, FYJ40 and FYJ41 were similarly obtained by transduction of strain K113 (Table 1).

\section{Measurement of $\beta$-galactosidase}

Mid-log phase cultures in LB, RB or minimal media (with or without supplementation with various carbon sources), were collected by centrifugation, washed twice with Z Buffer (Miller 1972) and assayed for $\beta$-galactosidase activity after lysis with sodium dodecyl sulfatechloroform (Miller 1972). The data were recorded in triplicate with no less than three independent experiments.

\section{Site-directed mutagenesis}

Site-directed mutagenesis was done as we recently described with little changes (Feng et al., 2014). The PCR reaction system $(25 \mu \mathrm{L})$ consisted of the following components: $2.5 \mu \mathrm{L}$ of $10 \times \mathrm{pfx}$ buffer (Invitrogen), $0.5 \mu \mathrm{L}$ of $40 \mathrm{mmol} / \mathrm{L}$ dNTP $\operatorname{mix}(10 \mathrm{mmol} / \mathrm{L}$ each), $1.0 \mu \mathrm{L}$ of forward/reverse primers (10 pmol/ $\mu \mathrm{L}), 1.0 \mu \mathrm{L}$ of $\mathrm{pET} 28$-fadRec as template $(5 \mathrm{ng} / \mu \mathrm{L}), 0.5 \mu \mathrm{L}$ of Platinum pfx (2.5 U/ $\mu \mathrm{L}$, Invitrogen), and $17.5 \mu \mathrm{L}$ of distilled sterilized $\mathrm{H}_{2} \mathrm{O}$. The reaction was performed using the program consisting of a denaturing cycle at $95^{\circ} \mathrm{C}$ for $5 \mathrm{~min} ; 20$ cycles comprised of $95^{\circ} \mathrm{C}$ for $50 \mathrm{~s}, 60^{\circ} \mathrm{C}$ for $50 \mathrm{~s}$, and $68^{\circ} \mathrm{C}$ for $6 \mathrm{~min}$ and a final step of $8 \mathrm{~min}$ at $68^{\circ} \mathrm{C}$. To remove the residual template plasmid pET28-fadRec, the gel purified $\mathrm{PCR}$ products were digested for $1 \mathrm{~h}$ with $\mathrm{Dpnl}(20 \mathrm{U} / \mu \mathrm{L}, \mathrm{NEB})$ at $37^{\circ} \mathrm{C}$. Subsequently, they were transformed into chemically-competent cells of $\mathrm{DH} 5 \alpha$ and the inserts of purified plasmids were verified by direct DNA sequencing. The plasmids were transformed into BL21 (Tuner) to produce the FadR mutant proteins.

\section{Protein expression and purification}

The hexahistidine-tagged E. coli FadR proteins were produced in E. coli BL21 (DE3) carrying the appropriate expression plasmids (e.g., pET28-fadRec, Table 1) by induction of bacterial cultures at 
Table 2. Primers used in this study

\begin{tabular}{|c|c|}
\hline Primers & Primer sequences $\left(5^{\prime}-3^{\prime}\right)$ \\
\hline lacZ-R & GACCATGATTACGGATTCACTG \\
\hline fadR_K113-F (BamHI) & CGGGATCCATGGTCATTAAGGCGCAAAGCC \\
\hline fadR_K113-R (Xhol) & CCGCTCGAGTTATCGCCСCTGAATGGCTAAATC \\
\hline fadL-FadR1-F & GCAACATTCCAGCTGGTCCGACCTATACTCTCGCC \\
\hline fadL-FadR1-R & GGCGAGAGTATAGGTCGGACCAGCTGGAATGTTGC \\
\hline fadL-FadR2-F & СTCTCGCСАCTGGTCTGATTTCTAAGATGTACCTC \\
\hline fadL-FadR2-R & GAGGTACATCTTAGAAATCAGACCAGTGGCGAGAG \\
\hline fadD-FadR1-F & GAAACAGCGGCTGGTCCGCTGTTTCTGCATTCT \\
\hline fadD-FadR1-R & AGAATGCAGAAACAGCGGACCAGCCGCTGTTTC \\
\hline fadD-FadR2-F & GTAATTATCAAGCTGGTATGATGAGTTAATATTATG \\
\hline fadD-FadR2-R & САTAATATTAACTCATCATACCAGCTTGATAATTAC \\
\hline fadBA-F & ACTTCGACTCATCTGGTACGACCAGATCACCTTGCGG \\
\hline fadBA-R & CCGCAAGGTGATCTGGTCGTACCAGATGAGTCGAAGT \\
\hline fabA-F & TTTATTCCGAACTGATCGGACTTGTTCAGCGTACACGTGTTAGCTATCCTGCGTGC \\
\hline$f a b A-R$ & GCACGCAGGATAGCTAACACGTGTACGCTGAACAAGTCCGATCAGTTCGGAATAAA \\
\hline fabB-F & TCTATTAAATGGCTGATCGGACTTGTTCGGCGTACAAGTGTACGCTATTGTGCATTC \\
\hline$f a b B-R$ & GAATGCACAATAGCGTACACTTGTACGCCGAACAAGTCCGATCAGCCATTTAATAGA \\
\hline F74G-F & CGACGAAGGTGAATAATGGCTGGGAAACTTCCGGTT \\
\hline F74G-R & AACCGGAAGTTTCCCAGCCATTATTCACCTTCGTCG \\
\hline W75G-F & CGAAGGTGAATAATTTCGGGGAAACTTCCGGTTTAAA \\
\hline W75G-R & TTTAAACCGGAAGTTTCCCCGAAATTATTCACCTTCG \\
\hline F74G/W75G-F & CGACGAAGGTGAATAATGGCGGGGAAACTTCCGGTTTAAA \\
\hline F74G/W75G-R & TTTAAACCGGAAGTTTCCCCGCCATTATTCACCTTCGTCG \\
\hline pBAD-chk5 & CTGTTTCTCCATACCCGTT \\
\hline pBAD-chk3 & GGCTGAAAATCTTCTCT \\
\hline fadRec-F2 (EcoRI) & AACCGAATTCATGGTCATTAAGGCGCAAAGCC \\
\hline fadRec-R2 (Sall) & CCGGTCGACTTATCGCCCCTGAATGGCTAAATC \\
\hline T7-F & TAATACGACTCACTATAGGG \\
\hline T7-R & GCTAGTTATTGCTCAGCGG \\
\hline
\end{tabular}

The sequences underlined are restriction sites, and the bold letters are putative FadR binding sites.

an $\mathrm{OD}_{600 \mathrm{~nm}}$ of $0.8-1.0$ with $0.3 \mathrm{mmol} / \mathrm{L} \mathrm{IPTG}$ at $30^{\circ} \mathrm{C}$ for $3 \mathrm{~h}$ (Feng \& Cronan, 2011b, Feng \& Cronan, 2010). The cells were pelleted, washed twice with ice-cold PBS buffer $\left(101.4 \mathrm{mmol} / \mathrm{L} \mathrm{Na} \mathrm{HPO}_{4}\right.$, $1.8 \mathrm{mmol} / \mathrm{L} \mathrm{KH} \mathrm{KH}_{2} \mathrm{PO}_{4}, 137 \mathrm{mmol} / \mathrm{L} \mathrm{NaCl}, 2.7 \mathrm{mmol} / \mathrm{L} \mathrm{KCl}, 8 \%$ glycerol, pH7.4), dissolved in the same buffer and lysed using a French Press. The extracts were centrifuged to remove bacterial debris and the supernatants loaded onto a nickel chelate column (Qiagen). Following washing with ten column volumes of PBS buffer containing $50 \mathrm{mmol} / \mathrm{L}$ imidazole, the FadR proteins were eluted with $150 \mathrm{mmol} / \mathrm{L}$ imidazole. Appropriate eluted protein fractions were pooled and dialyzed against PBS buffer, then concentrated by ultrafiltration (30 kDa cut-off, Amicon Ultra) (Feng \& Cronan, 2010). The protein purity was judged by $12 \%$ SDS-PAGE, followed by staining with Coomassie brilliant blue R250 (Sigma, St. Louis, MO).
Electrophoretic mobility shift assays

Electrophoretic mobility shift assays were performed to address interaction between FadR and series of taget DNA probes (e.g., fadD and fadL (Feng \& Cronan, 2012)) essentially as previously reported (Feng \& Cronan, 2011b, Feng \& Cronan, 2011a, Feng \& Cronan, 2009b). With an exception of fadM probe (PCR products) (Feng \& Cronan, 2009b), all of the FadR-recognizable probes were prepared by annealing two complementary primers (Table 2 ) by incubation in TEN buffer $(10 \mathrm{mmol} / \mathrm{L}$ Tris- $\mathrm{HCl}, 1 \mathrm{mmol} / \mathrm{L}$ EDTA, $100 \mathrm{mmol} / \mathrm{L} \mathrm{NaCl}, \mathrm{pH} \mathrm{8.0)}$ at $95^{\circ} \mathrm{C}$ for $5 \mathrm{~min}$ followed by slow cooling to $25^{\circ} \mathrm{C}$ and then digoxigenin (DIG) labeling by terminal transferase with DIG-ddUTP (Roche). The DIG-labeled DNA probes (either $0.1 \mathrm{pmol}$ or $0.2 \mathrm{pmol}$ ) were incubated with either DNA binding protein in binding buffer (Roche) for $15 \mathrm{~min}$ at room temperature and then analyzed by native PAGE (7-7.5\% PAGE for all probes). 


\section{Bioinformatic analyses}

The known FadR sequences were all from the NCBI database and the multiple alignments were done using ClustalW2 (http://www.ebi. ac.uk/Tools/clustalw2/index.html), and the resultant output was processed by program ESPript 2.2 (http://espript.ibcp.fr/ESPript/cgibin/ESPript.cgi), generating the final BLAST version. The FadR structure was visualized using PyMol software.

\section{ACKNOWLEDGEMENTS}

This work was supported by a start-up package from Zhejiang University (YF). YF is a recipient of the "Young 1000 Talents" award. We would like to thank Dr. Zhi Li (Department of Biochemistry, UIUC) for helpful discussion on E. coli FadR structure.

\section{COMPLIANCE WITH ETHICS GUIDELINES}

Yongchang Zhang, Rongsui Gao, Huiyan Ye, Qingjing Wang and Youjun Feng declare that they have no conflict of interest.

This article does not contain any studies with human or animal subjects performed by the any of the authors.

\section{OPEN ACCESS}

This article is distributed under the terms of the Creative Commons Attribution License which permits any use, distribution, and reproduction in any medium, provided the original author(s) and the source are credited.

\section{REFERENCES}

Campbell JW, Cronan JE Jr (2001) Escherichia coli FadR positively regulates transcription of the $\mathrm{fabB}$ fatty acid biosynthetic gene. J Bacteriol 183:5982-5990

Clark D (1981) Regulation of fatty acid degradation in Escherichia coli: analysis by operon fusion. J Bacteriol 148:521-526

Clark D, Cronan J (2005) Two-carbon compounds and fatty acids as carbon sources. In: Bock A, Curtiss III R, Karper J, Karp P, Neidhardt F, Nystrom T, Slauch J, Squiress C, Ussery D (eds) EcoSal-Escherichia coli and Salmonella: cellular and molecular biology. http://www.ecosal.org. ASM Press, Washington, DC

Clark DP, DeMendoza D, Polacco ML, Cronan JE Jr (1983) Betahydroxydecanoyl thio ester dehydrase does not catalyze a ratelimiting step in Escherichia coli unsaturated fatty acid synthesis. Biochemistry 22:5897-5902

Cronan JE Jr (1997) In vivo evidence that acyl coenzyme A regulates DNA binding by the Escherichia coli FadR global transcription factor. J Bacteriol 179:1819-1823

Cronan JE Jr, Subrahmanyam S (1998) FadR, transcriptional coordination of metabolic expediency. Mol Microbiol 29:937-943

DiRusso CC, Heimert TL, Metzger AK (1992) Characterization of FadR, a global transcriptional regulator of fatty acid metabolism in Escherichia coli. Interaction with the $f a d B$ promoter is prevented by long chain fatty acyl coenzyme A. J Biol Chem 267:8685-8691

DiRusso CC, Metzger AK, Heimert TL (1993) Regulation of transcription of genes required for fatty acid transport and unsaturated fatty acid biosynthesis in Escherichia coli by FadR. Mol Microbiol 7:311-322

DiRusso CC, Tsvetnitsky V, Hojrup P, Knudsen J (1998) Fatty acylCoA binding domain of the transcription factor FadR. Characterization by deletion, affinity labeling, and isothermal titration calorimetry. J Biol Chem 273:33652-33659

Farewell A, Kvint K, Nystrom T (1998) Negative regulation by RpoS: a case of sigma factor competition. Mol Microbiol 29:1039-1051

Feng Y, Cronan JE (2009a) Escherichia coli unsaturated fatty acid synthesis: complex transcription of the fabA gene and in vivo identification of the essential reaction catalyzed by FabB. J Biol Chem 284:29526-29535

Feng Y, Cronan JE (2009b) A new member of the Escherichia coli fad regulon: transcriptional regulation of fadM (ybaW). J Bacteriol 191:6320-6328

Feng Y, Cronan JE (2010) Overlapping repressor binding sites result in additive regulation of Escherichia coli FadH by FadR and ArcA. J Bacteriol 192:4289-4299

Feng Y, Cronan JE (2011a) Complex binding of the FabR repressor of bacterial unsaturated fatty acid biosynthesis to its cognate promoters. Mol Microbiol 80:195-218

Feng Y, Cronan JE (2011b) The Vibrio cholerae fatty acid regulatory protein, FadR, represses transcription of $p / s B$, the gene encoding the first enzyme of membrane phospholipid biosynthesis. Mol Microbiol 81:1020-1033

Feng Y, Cronan JE (2012) Crosstalk of Escherichia coli FadR with global regulators in expression of fatty acid transport genes. PloS one $7: \mathrm{e} 46275$

Feng Y, Li M, Zhang H, Zheng B, Han H, Wang C, Yan J, Tang J, Gao GF (2008) Functional definition and global regulation of Zur, a zinc uptake regulator in a Streptococcus suis serotype 2 strain causing streptococcal toxic shock syndrome. J Bacteriol 190:7567-7578

Feng Y, Napier BA, Manandhar M, Henke SK, Weiss DS, Cronan JE (2014) A Francisella virulence factor catalyses an essential reaction of biotin synthesis. Mol Microbiol 91:300-314

Gui L, Sunnarborg A, LaPorte DC (1996) Regulated expression of a repressor protein: FadR activates ic/R. J Bacteriol 178:47044709

Guzman LM, Belin D, Carson MJ, Beckwith J (1995) Tight regulation, modulation, and high-level expression by vectors containing the arabinose PBAD promoter. J Bacteriol 177:41214130

Haldimann A, Wanner BL (2001) Conditional-replication, integration, excision, and retrieval plasmid-host systems for gene structurefunction studies of bacteria. J Bacteriol 183:6384-6393

He XY, Yang SY, Schulz H (1997) Cloning and expression of the $\mathrm{fad} H$ gene and characterization of the gene product 2,4-dienoyl coenzyme A reductase from Escherichia coli. Eur J Biochem 248:516-520

Henry MF, Cronan JE Jr (1992) A new mechanism of transcriptional regulation: release of an activator triggered by small molecule binding. Cell 70:671-679

Hughes KT, Simons RW, Nunn WD (1988) Regulation of fatty acid degradation in Escherichia coli: fadR superrepressor mutants are unable to utilize fatty acids as the sole carbon source. J Bacteriol 170:1666-1671 
Iram SH, Cronan JE (2005) Unexpected functional diversity among FadR fatty acid transcriptional regulatory proteins. J Biol Chem 280:32148-32156

Iram SH, Cronan JE (2006) The beta-oxidation systems of Escherichia coli and Salmonella enterica are not functionally equivalent. J Bacteriol 188:599-608

Maloy SR, Nunn WD (1981) Role of gene fadR in Escherichia coli acetate metabolism. J Bacteriol 148:83-90

Miller JH (1972) Experiments in molecular genetics. Cold Spring Harbor Laboratory Press, Cold Spring Harbor

Miller JH (1992) A short course in bacterial genetics. Cold Spring Harbor Laboratory, Cold Spring Harbor
Nunn WD, Giffin K, Clark D, Cronan JE Jr (1983) Role for fadR in unsaturated fatty acid biosynthesis in Escherichia coli. J Bacteriol 154:554-560

van Aalten DM, DiRusso CC, Knudsen J, Wierenga RK (2000) Crystal structure of FadR, a fatty acid-responsive transcription factor with a novel acyl coenzyme A-binding fold. Embo J 19:5167-5177

van Aalten DM, DiRusso CC, Knudsen J (2001) The structural basis of acyl coenzyme A-dependent regulation of the transcription factor FadR. Embo J 20:2041-2050

Xu Y, Heath RJ, Li Z, Rock CO, White SW (2001) The FadR.DNA complex. transcriptional control of fatty acid metabolism in Escherichia coli. J Biol Chem 276:17373-17379 